\title{
APLICACIÓN E IMPACTO DE LAS HERRAMIENTAS SOCIALES DEL INTERNET EN LA COMUNICACIÓN Y PROCESO DE INTERAPRENDIZAJE EN LA FACULTAD DE CIENCIAS SOCIALES Y COMUNICACIÓN
}

Autores:

Verónica López Y. ${ }^{1}$ Paola Calderón A. ${ }^{1}$ Patricia Castañeda' 



\section{Resumen}

Las herramientas sociales del internet, se han convertido en protagonistas cuando hablamos de formas de interacción y de comunicación, es así, que su utilización se ha transformado en un motor fundamental para diversas áreas del conocimiento y del esparcimiento humano. Sin lugar a dudas, herramientas como los blogs, el youtube, el facebook, han trascendido, llegando también a ser importantes para el desarrollo y principalmente para la transformación de la Educación Superior. Por esta razón, se ha tomado el tema como punto de partida para realizar la presente investigación en donde se analiza el uso y el impacto que tienen dichas herramientas sociales en los principales actores del proceso de interaprendizaje de la Facultad de Ciencias Sociales y Comunicación de la Universidad Tecnológica Equinoccial a nivel nacional.

La investigación ha sido enriquecida con datos recopilados a través de entrevistas y de encuestas a estudiantes, docentes y profesionales expertos en áreas afines al tema planteado; otra de las motivaciones que generó este trabajo, fue el encontrar datos de otros países que ya manejan oficialmente las herramientas sociales dentro de sus ámbitos académicos frente a la falta de documentación que registra el Ecuador en esta área, de aquí se genera también un análisis profundo del uso que realizan de estas herramientas otras Universidades del País.

Se pudo determinar que las herramientas están asociadas con el entretenimiento y la diversión, pero tienen un fuerte potencial para la mejora de la comunicación y de la educación. Se concluye que si bien las herramientas sociales del Internet son conocidas y utilizadas por la mayoría de estudiantes y docentes, es necesario un aprendizaje previo a su utilización en el ámbito educativo.

Palabras clave: Herramientas sociales del Internet, educación, interaprendizaje.

Recibido: Mayo 2012

Aprobado: Noviembre 2012

129 


\section{ABSTRACT}

The internet social tools, have become actors when it comes to forms of interaction and communication, so that its use has become a key driver for various areas of knowledge and human recreation. Undoubtedly, tools like blogs, youtube, facebook, have transcended and on to be important mainly for development and transformation of higher education. For this reason, the issue has been taken as a starting point for this research which analyzes the use and impact of these social tools in the main actors in the process of mutual learning in Faculty of Social Sciences and Communication - Technology Equator University.

Research has been enriched with data collected through interviews and surveys of students, teachers and experts in areas related to the topic raised, one of the reasons that led to this work, was to find data from other countries that officially handled social tools within their academic address the lack of documentation that records Ecuador in this area, hence it also generates a deep analysis of the use of these tools perform other universities.

It was determined that the tools are associated with entertainment and fun, but they have a strong potential to improve communication and education. We conclude that although the Internet social tools are known and used by the majority of students and teachers, learning necessary prior to its use in education.

Keywords: Internet Social tools, education, shared learning. 


\section{Introducción}

"En el contexto de la sociedad del conocimiento, las tecnologías de uso educativo ya sean empleadas para la enseñanza presencial o a distancia se han convertido en un soporte fundamental para la instrucción, beneficiando a un universo cada vez más amplio de personas. Esta asociación entre tecnología y educación no sólo genera mejoras de carácter cuantitativo es decir, la posibilidad de enseñar a más estudiantes, sino que principalmente de orden cualitativo: los educandos encuentran en Internet nuevos recursos y posibilidades de enriquecer su proceso de aprendizaje”. (Romani, 2007:15)

La vinculación entre la tecnología y la educación, genera para los docentes mejoras cuantitativas (inter-enseñar a más estudiantes), pero principalmente cualitativas, cuando los educandos encuentran en Internet nuevos recursos y posibilidades de enriquecer su proceso de aprendizaje. Las herramientas se convierten en una opción para evitar la desvinculación y el aislamiento entre los actores del proceso pedagógico de interaprendizaje y permiten la creación de propios espacios académicos.

En las últimas décadas se ha podido constatar un cambio vertiginoso en la relación de la sociedad con las tecnologías digitales. El Internet se ha convertido en el medio de comunicación más extendido a nivel mundial ya que permite establecer la cooperación y colaboración entre gran número de comunidades y grupos de interés, distribuidos alrededor del mundo. Dentro de este contexto aparece la Web 2.0 (Web de segunda generación o Web social) que se presenta como una web amigable y de fácil difusión en ámbitos diversos y cotidianos como políticos, laborales, económicos, mercadológicos y el de interés para este proyecto, el ámbito de la educación; en donde el modelo educativo tradicional en el que el profesor es el único encargado de transmitir el conocimiento y el alumno un ente pasivo, ha dado paso a una transformación en la que los estudiantes tienen la capacidad de utilizar las herramientas sociales del internet como por ejemplo, blogs, foros, Facebook, Wikis, Twitter, Flickr, etc., para manejar todo tipo de información, sintetizarla y compartirla, generando nuevos métodos de enseñanza y técnicas de aprendizaje de manera interactiva.

"El concepto de intercreatividad se aproxima, desde una perspectiva tecnosocial, al potencial colaborativo que está tras el uso de las tecnologías en red, ya que lo que se construye es un ciberespacio para compartir el conocimiento entre personas, a través de Networks de cooperación recíproca.”(Romani, 2007:15)

La importancia del uso de las herramientas del Internet es la oportunidad de la generación del conocimiento con la participación activa de docentes y estudiantes a través de la red desarrollando la capacidad creativa por medio de la comunicación de ideas basadas en conocimientos previos e investigación.

Como docentes nos hemos dado cuenta que existe un incremento del uso de estas herramientas sociales del Internet por parte de estudiantes y docentes. Cabe resaltar que en gran medida los actores del proceso de interaprendizaje relacionan a estas herramientas con el entretenimiento. Sin embargo la comunicación es un eje transversal que existe y puede colaborar para mejorar el proceso de interaprendizaje en el ámbito educativo. Por estos motivos se decidió realizar una investigación cuyo objetivo principal es conocer la aplicación y el impacto potencial que tienen las herramientas sociales del Internet en el proceso de comunicación en el interaprendizaje. Este estudio es realizado en la Facultad de Ciencias Sociales y Comunicación de la Universidad por medio del análisis y determinación del uso de las herramientas del Internet en la comunicación dentro del ámbito educativo a través de la visita de portales web de varias universidades del país, entrevistas a profesionales, entrevistas a docentes de la Universidad y encuestas a los estudiantes.

Como hipótesis se planteó que las herramientas informáticas sociales de segunda generación impulsan el mejoramiento del proceso de aprendizaje. 


\section{Metodología}

Entre las técnicas que se utilizaron para la recolección de datos, se pueden citar:

- Observación directa

- Encuesta en sus dos modalidades: entrevista y cuestionario

- El análisis documental y análisis de contenido.

Primeramente, para la Investigación se realizó un análisis al uso de herramientas sociales del Internet en varias Universidades del país. Para esto se tomó una muestra de 35 páginas Web de universidades del Ecuador, las mismas que fueron seleccionadas por el Rankin Web de las Universidades del mundo' .

\section{LISTA DE UNIVERSIDADES}

1. Escuela Politécnica Superior del Litoral

2. Universidad Del Azuay

3. Escuela Politécnica Nacional

4. Universidad Católica Del Ecuador

5. FLACSO

6. Escuela Superior Politécnica Del Chimborazo

7. UNIVERSIDAD DE CUENCA

8. Universidad Politécnica Salesiana

9. Escuela Politécnica Del Ejército

10. Universidad Central Del Ecuador

11. Universidad Tecnológica Equinoccial

12. Universidad Nacional De Loja

13. Universidad Estatal De Milagro

14. Universidad Técnica Del Norte

15. Universidad Internacional Sek

16. Universidad Nacional De Chimborazo

17. Universidad Del Pacífico

18. Universidad De Guayaquil

19. Universidad Católica De Santiago De Guayaquil
20. Instituto De Altos Estudios Nacionales

21. Universidad Técnica De Ambato

22. Universidad Tecnológica América

23. Universidad De Especialidades Espíritu Santo

24. Universidad Santa María De Chile, Campus Guayaquil

25. Universidad De Las Américas

26. Universidad De Especialidades Turísticas

27. Universidad Estatal De Bolívar Ranking Mundial

28. Universidad Particular Sek

29. Universidad Técnica Estatal De Quevedo

30. Universidad Internacional Del Ecuador

31. Universidad Metropolitana Del Ecuador

32. Universidad Técnica De Manabí

33. Pontificia Universidad Católica Del Ecuador Sede Ibarra

34. Universidad Laica Eloy Alfaro De Manabí

35. Universidad Técnica De Cotopaxi

\section{ENTREVISTAS A PROFESIONALES EXPERTOS}

También se realizó una investigación con profesionales del área, para conocer sus opiniones y la experiencia que han tenido con las herramientas sociales del Internet en la comunicación, en los procesos de interaprendizaje y en el ámbito laboral. En este punto colaboraron el Ing. Fabián Jaramillo y Msc. Karina Zela, docentes de la ESPE y la Msc. Gabriela Coronel, docente de la UTPL.

\section{ENTREVISTAS A DOCENTES Y ESTUDIANTES}

Además la investigación fue realizada a docentes y estudiantes de la Facultad de Ciencias Sociales y Comunicación en los tres Campus de la Universidad Tecnológica Equinoccial: Quito, Santo Domingo y Salinas; los mismos que brindaron información acerca de temas como: impacto y aplicación del Internet en la educación, las herramientas de nueva tecnología conocidas y utilizadas para mejorar la comunicación en el proceso de interaprendizaje y las redes sociales.

1 Ranking Web of Universities. http://www.webometrics.info/about_es.html. Visitada en junio 2010 
Para el universo se tomó como referencia corresponde al total de estudiantes matriculados para el período octubre-2009 marzo-2010, en la Facultad de Ciencias Sociales y Comunicación en toda la Universidad.

A continuación una tabla con el detalle del universo en cada campus, especificando estudiantes y docentes:

\begin{tabular}{|l|c|c|}
\hline \multicolumn{1}{|c|}{ CAMPUS } & ESTUDIANTES & DOCENTES \\
\hline QUITO & 790 & 110 \\
\hline SALINAS & 86 & 6 \\
\hline SANTO DOMINGO & 240 & 9 \\
\hline TOTAL & 1116 & 125 \\
\hline
\end{tabular}

TABLA 1: UNIVERSO DE ESTUDIO POR CAMPUS

\begin{tabular}{|l|l|}
\hline MUESTRA DOCENTES & 112 unidades de investigación \\
\hline MUESTRA ESTUDIANTES & 285 unidades de investigación \\
\hline
\end{tabular}

TABLA 2: MUESTRA DE ESTUDIO POR CAMPUS

En el Campus Quito la muestra de estudiantes se subdivide de acuerdo a las Carreras de la Facultad, lo que no sucede en Salinas y Santo Domingo porque la Facultad de Ciencias Sociales y Comunicación cuenta únicamente con la Carrera de Diseño Gráfico Publicitario en estos Campus.

Las unidades de investigación fueron seleccionadas de forma aleatoria simple.

Se realizó además un análisis sobre la forma en la que las herramientas sociales del Internet están siendo utilizadas por diferentes universidades del país. Para esto se tomó una muestra de 38 páginas Web de universidades del Ecuador, las mismas que fueron seleccionadas por el ranking web de universidades del mundo. (http:// www.webometrics.info/about_es.html). También se realizó una investigación con profesionales del área, para conocer sus opiniones y la experiencia que han tenido con las herramientas sociales del Internet en la comunicación, en los procesos de interaprendizaje y en el ámbito laboral.
Entre las técnicas que se utilizaron para la recolección de datos, se pueden citar:

- Observación directa

- Encuesta en sus dos modalidades: entrevista y cuestionario

- El análisis documental y análisis de contenido.

Los instrumentos utilizados fueron formatos de cuestionarios y guías de entrevistas:

\section{GUÍA DE ENTREVISTA DIRIGIDA A PROFESIONALES DEL ÁREA}

Tuvo por objeto conocer las opiniones de los profesionales y sus experiencias sobre aspectos relacionados con la utilización de herramientas sociales en los procesos de Inter-aprendizaje, en la comunicación y el aspecto laboral. Se trabajó con una investigación documental que permitió obtener y analizar datos provenientes de material bibliográfico y de la Web; la investigación de campo permitió recolectar los datos de las fuentes directas (docentes, estudiantes y profesionales del área).

\section{Resultados}

Sin lugar a dudas la educación debe adaptarse al cambiante entorno global y planificar estrategias que permitan a los estudiantes responder efectiva y eficientemente a los requerimientos económicos, sociales y humanos de la realidad a la que se enfrentan.

El estudio formula un nuevo reto para la educación superior, eliminar la brecha generacional que existe entre docentes y estudiantes, acercarnos a ellos con herramientas que potencialmente bien utilizadas desarrollarán en los educandos competencias para aprender toda la vida. Si lo logramos, nuestros estudiantes volverán a encontrar diversión en la incansable e inagotable tarea de aprender. 


\section{Universidades}

\section{Aprendizajes de análisis de las páginas web de las universidades según el ranking mundial ${ }^{2}$.}

Las líneas específicas de investigación del Raking incluyen, entre otras:

- Desarrollo de indicadores Web aplicados a los escenarios de I+D+l español, europeo, iberoamericano y mundial

- Estudios cuantitativos sobre la comunicación científica a través de revistas electrónicas y depósitos de documentos y el impacto de iniciativas del tipo Open Access.

- Desarrollo de indicadores sobre contenidos en la Sociedad de la Información

- Visualización de indicadores y redes sociales en la Web con interfaces gráficos amigables, dinámicos e interactivos

- Diseño y evaluación de técnicas de análisis documental de recursos Web

- Estudios de género aplicados a la actividad académica en la Web

- Desarrollo de técnicas de cibermetría aplicada basada en el posicionamiento en motores de búsqueda de sedes Web

- Análisis de consumo de información mediante minería de datos Web de ficheros log.

Estos indicadores permitieron el análisis de las universidades mencionadas en el apartado anterior, siendo los tres principales aprendizajes:

1. Las universidades incluyen en sus páginas web los íconos desde donde se puede acceder a las diferentes redes sociales.

2. Las principales redes sociales incluidas por las universidades son: facebook, twitter, youtube y en menor grado linkedin y flicker.

3. Se mantiene la funcionalidad de las redes en cuanto al 2 Información analizada del Ranking Mundial de Universidades en la Web. Iniciativa del Laboratorio de Cibermetría, que pertenece al CSIC, el mayor centro nacional de investigación de España. seguimiento social; sin embargo, no se visualiza una interacción que contribuya al proceso de aprendizaje.

\section{Entrevistas a los profesionales}

Los profesionales del área coincidieron en que la instituciones educativas deben actualizar su infraestructura tecnológica y capacitar a los docentes sobre el uso de la tecnología como herramienta pedagógica. "La tecnología no es una cosa mágica que mejorará la educación" (Ing. Fabián Jaramillo) sino su integración en el aula. Las redes sociales rompen barreras geográficas, temporales, generacionales, ahorran recursos y fomentan el aprendizaje colaborativo; facilitan la formación de comunidades de aprendizaje alrededor de temas diversos en los ámbitos: personal, educativo y profesional; mejoran la comunicación, la socialización, las relaciones interpersonales. Fueron creadas para fortalecer la opinión autónoma y responsable, y su principal problema es el abuso de esta libertad, por lo que es necesario formar íntegramente al ser humano que utilizará esa tecnología.

Las herramientas sociales en el entorno educativo deben ser consideradas desde el punto de vista pedagógico; el docente, participante activo del proceso de inter-aprendizaje debe cambiar sus paradigmas tradicionales por prácticas pedagógicas que fomenten el análisis, la síntesis y la reflexión. Con datos cuantitativos la presente investigación afirma que es ahí donde están nuestros estudiantes y podemos usar las redes sociales como aliadas en el campo educativo, lo importante es conversar y unir conceptos irreconciliables, hasta ahora, para los jóvenes trabajo-responsabilidad y placer-diversión. El cambio de paradigmas puede asustarnos o convertirse en un nuevo reto personal y profesional.

La educación en el Ecuador debe mejorar, existe un divorcio entre la Universidad y la sociedad, la falencia en la investigación mantiene al Ecuador como un país rico lleno de pobreza. Es la educación la alternativa para acortar esta brecha. Grandes pasos se 
van dando pero es un esfuerzo cuyos resultados se verán a largo plazo. “Aprender haciendo ese es el camino” (Mst. Karina Zela).

La sociedad de la información y el conocimiento demanda en estudiantes, docentes y profesionales, el desarrollo de la competencia de manejo de información.

La competencia en el manejo de información logrará, con la ayuda de las redes sociales, a convertirnos de consumidor de información a prosumidores críticos y reflexivos. Pero es importante diferenciar los tipos de información a los que estamos expuestos, es decir, esta competencia debe también desarrollar en nosotros la capacidad de seleccionar fuentes confiables de información.

\section{DOCENTES}

\section{Aprendizaje de las entrevistas a los docentes}

Los docentes tienen dominio en el manejo del Internet, además del tiempo de utilización, han realizado cursos específicos de búsqueda de información y de navegación en la Web.

Los docentes utilizan frecuentemente el Internet para realizar sus tareas de docencia. Las razones para su utilización son:

- Plataformas versátiles a la hora de investigar e impartir información.

- Se realiza investigación y retroalimentación.

- Es una forma de mantenerse actualizado.

El Internet es utilizado como recurso didáctico para la enseñanza, se considera importante para las tareas de docencia sobre todo por la actualidad de la información.

Los docentes consideran que el uso del Internet es muy importante para el futuro de las carreras profesionales de los estudiantes. Consideran además que en la actualidad es una herramienta fundamental para el desarrollo profesional y porque la comunicación que es la base social, académica y profesional, fluye con mayor rapidez.

Se considera que la Plataforma Virtual de la UTE facilita el trabajo de docencia, porque permite subir y acceder a información específica, permite la planificación académica y porque las múltiples herramientas permiten la interrelación con los estudiantes.

Los docentes definen a la comunicación virtual como la forma para poder comunicar conceptos, teorías, discutir y dialogar sobre diferentes temas en cualquier parte del mundo y a cualquier hora.También se considera como una manera de interactuar con el estudiante mediante un equipo tecnológico.

Entre las herramientas sociales del Internet que los docentes conocen están:

- Wikie

- Páginas de inicio personalizadas

- Redes sociales personales

- Compartir videos

- Sistemas de Creación y Alojamiento

Las herramientas que han utilizado son:

- Blogger

- Wikipedia

- Wikimedia

- Gmail

- Google

- Windows live

- Flickr

- Youtube

- Facebook

Los docentes afirman haber recurrido a las herramientas mencionadas en el punto anterior para realizar las tareas de docencia 
y además consideran que dichas herramientas han mejorado la comunicación con los estudiantes.

Se considera necesario recurrir a las herramientas del Internet como fuentes de investigación y de comunicación para mejorar el proceso de inter-aprendizaje; una de las causas que se menciona para utilizar como fuente de investigación, es la falta de accesibilidad a libros.

En cuanto a capacitación para el uso y aplicación de herramientas de Internet para la docencia, no se considera necesaria, excepto cuando los docentes no tienen un nivel óptimo para el manejo adecuado.

Es necesario elevar el nivel de la utilización de las herramientas de Internet para la educación y la comunicación con los estudiantes, además se considera necesario la inclusión de dichas herramientas en una plataforma virtual.

Los docentes creen que es factible incluir las herramientas sociales del Internet en sus asignaturas.

El enriquecimiento educativo personal con base en experiencias educativas y profesionales ajenas es considerado muy importe porque permite el intercambio de opiniones, porque es la base del conocimiento y porque sirve como experiencia para la futura aplicación en el área laboral.

Por último, se considera que la interactividad sí genera conocimiento porque hace más activos a los estudiantes, además porque se cree que la experiencia ayuda a adquirir conocimiento.

\section{ESTUDIANTES}

\section{Aprendizajes de las encuestas a los estudiantes}

\section{Evaluar el uso del internet en el aprendizaje}

Uso del internet como recurso de estudio

El auge de las de las herramientas sociales del Internet, permite a los estudiantes profundizar sus conocimientos.

\begin{tabular}{|l|r|r|}
\hline Carrera & \multicolumn{1}{|c|}{ Frecuencia } & Porcentaje \\
\hline Diseño Gráfico Publicitario & 72 & $64 \%$ \\
\hline Publicidad Y Gestión & 24 & $21 \%$ \\
\hline Relaciones Públicas & 10 & $9 \%$ \\
\hline Periodismo & 2 & $2 \%$ \\
\hline Educación Inicial & 5 & $4 \%$ \\
\hline & $\mathbf{1 1 3}$ & $\mathbf{1 0 0 \%}$ \\
\hline
\end{tabular}

TABLA 3: USO DEL INTERNET EN EL APRENDIZAJE

En cuanto al proceso de interaprendizaje el estudio afirma que los estudiantes en general utilizan al Internet como herramienta de estudio para profundizar sus conocimientos. El 34\% de estudiantes de Publicidad y Gestión (24 de 70 estudiantes), el 25\% de estudiantes de Periodismo (2 de 8 estudiantes), el 37\% de estudiantes de Diseño Gráfico Publicitario (27 de 72 estudiantes), el 37\% de estudiantes de Relaciones Públicas (10 de 27 estudiantes) y el $45 \%$ de estudiantes de Educación Inicial (5 de 11 estudiantes) utilizan casi siempre el Internet como recurso de estudio. El resto de estudiantes lo utilizan para entretenimiento y comunicación. En general los estudiantes de Diseño, Publicidad y Educación Inicial se conectan al Internet en un promedio de 1 a dos horas y los estudiantes de Relaciones públicas y Periodismo de 3 a 4 horas. 


\begin{tabular}{|l|r|r|r|}
\hline Carrera & Frecuencia & Porcentaje & Porcentaje acumulado \\
\hline Diseño Gráfico Publicitario & 106 & $59 \%$ & $59 \%$ \\
\hline Publicidad Y Gestión & 46 & $26 \%$ & $85 \%$ \\
\hline Relaciones Públicas & 17 & $9 \%$ & $94 \%$ \\
\hline Periodismo & 6 & $3 \%$ & $97 \%$ \\
\hline Educación Inicial & 6 & $3 \%$ & $100 \%$ \\
\hline & $\mathbf{1 8 1}$ & $\mathbf{1 0 0} \%$ & \\
\hline
\end{tabular}

TABLA 4: USO DEL INTERNET COMO MEDIO DE COMUNICACIÓN Y ENTRETENIMIENTO

Si bien las razones varían el estudio nos permite evidenciar que los estudiantes tienen entre sus prioridades el entretenimiento, la comunicación por chat y en tercer lugar la búsqueda de información. Las redes sociales que utilizan dependen del entorno social y académico; la razón fundamental de uso la comunicación dinámica y la elección depende de las actividades que estén realizando (entretenimiento, comunicación o búsqueda de información).

\section{Discusión}

En los estudiantes se encontró una gran disposición por utilizar estas herramientas en el proceso educativo, a pesar que en su mayoría utilizan estas herramientas para la comunicación y distracción, opinan que es una necesidad en la actualidad. Los docentes en general manifiestan su interés por el uso de estas herramientas en el proceso, sin embargo también existe una desconfianza en su uso en el área educacional, debido principalmente a que estas herramientas las relacionan con entretenimiento y distracción.

\section{Conclusiones y recomendaciones}

A partir de la investigación teórica, observando la situación actual del proceso y con base en las conclusiones de la investigación de campo, podemos comprobar la hipótesis del proyecto: "Las herramientas informáticas sociales del Internet, impulsan el mejoramiento del proceso de aprendizaje". No obstante, para poder comprobar dicha hipótesis se cree que es necesario la participación de docentes y estudiantes en el manejo de herramientas sociales, concluyendo que es imprescindible la capacitación previa a los docentes sobre el uso, manejo y aplicación de estas herramientas. El docente como motivador en clases puede proponer a los estudiantes utilizar estas herramientas para mejorar el proceso, de esta forma con una capacitación sobre el tema, docentes podrán utilizar de manera más segura y permanente las herramientas sociales en sus clases, motivando al estudiante y generando una comunicación recíproca. Habiendo ya realizado un primer curso sobre capacitación de herramientas sociales del Internet como parte de la difusión de los resultados, se tuvo una respuesta positiva de docentes a colaborar con procesos relacionados con el uso de herramientas sociales del Internet en la Educación.

Además una de las participantes del proyecto de investigación, utilizó una red social como metodología principal en una de sus clases, obteniendo resultados positivos en evaluaciones y resultados del aprendizaje.

Para terminar primeramente recomendamos la necesaria capacitación a docentes sobre el uso y aplicación de las herramientas sociales del Internet en el ámbito de comunicación dentro de la educación, después recomendamos la activación del sitio Web para la Facultad de Ciencias Sociales y Comunicación en donde docentes y estudiantes tengan la posibilidad de publicar y transmitir información con el objetivo de mejorar el proceso de aprendizaje e incentivar a la generación de conocimiento.

La participación del uso de estas herramientas no puede ser explotada en su totalidad en el proceso pedagógico sin la guía, motivación y promoción por parte de los docentes, por este motivo para conseguir la participación por medio de herramientas sociales de internet se necesita una capacitación previa. 


\section{Bibliografía}

Blood, R. (2007). Universo del Weblog. Ediciones Gestión 2000, Barcelona-España, 83

Castells, Manuel. La transición a la Sociedad Red.2007, 125,197

Cobo Romaní, C. y Pardo Kuklinski, H. (2007). Planeta Web 2.0. Inteligencia colectiva o medios Fast food. Group de Recerca d'Interaccions Digitals, Universitat de Vic. FLACSO. Barcelona / México DF. , 27

García, A. (2004). Las nuevas tecnologías en la enseñanza. Akal Ediciones, Madrid-España. 15

Guayasamin, C. (2004). Internet y la investigación científica. Magisterio Español, España, 26

Litwin, E. (2005). Tecnologías educativas en tiempos de Internet. Amorrortu Editores, Buenos Aires - Madrid.60

Monsoriu, M. (2008). Manual de Redes Sociales en Internet. Madrid: Primera Edición. Creaciones Copyright, 2009,82

Terán, G. (2006). El Proyecto de Investigación: Cómo elaborar. Departamento de Investigación y Doctrina ESMIL. Ecuador. 